\title{
Bioactivity guided isolation of apigenin from Stachys lavandulifolia Vahl. in mice with anxiolytic effects
}

\author{
Mohammad Rabbani ${ }^{1}$, Seyed-Ebrahim Sajjadi ${ }^{2}$, Masoumeh Karimi-Firouzjaei ${ }^{1}$, and Mustafa Ghanadian ${ }^{2,3^{*}}$ \\ ${ }^{1}$ Department of Pharmacology, Isfahan University of Medical Sciences, Isfahan, Iran \\ ${ }^{2}$ Department of Pharmacognosy, School of Pharmacy and Pharmaceutical Sciences, Isfahan University of Medical Sciences, Isfahan, Iran \\ ${ }^{3}$ Isfahan Pharmaceutical Sciences Research Centre, School of Pharmacy and Pharmaceutical Sciences, Isfahan University of Medical Sciences, Isfahan,
} Iran

\section{A R T I C L E I N F 0}

Article Type:

Original Article

\section{Article History:}

Received: 7 October 2017

Accepted: 4 February 2018

\section{Keywords:}

Anxiety

Stachys lavandulifolia Vahl. Elevated

plus-maze

Apigenin

\begin{abstract}
A B S T RA C T
Introduction: Stachys lavandulifolia is traditionally used for the treatment of anxiety. Our previous study showed that the ethyl acetate fraction of the plant had substantial anxiolytic action in mice. The present study was aimed to investigate the main constituent responsible for the observed effects.

Methods: Ethyl acetate extract was fractionated using column chromatography. Yielded fractions (FR1-4) at $50 \mathrm{mg} / \mathrm{kg}$, and diazepam at $0.5 \mathrm{mg} / \mathrm{kg}$ were tested on the elevated plus-maze (EPM). Bioactive fraction was subjected to more purification on repeated chromatography columns. The isolated compound was identified based on ${ }^{1} \mathrm{H}-\mathrm{NMR},{ }^{13} \mathrm{C}-\mathrm{NMR}$, and ESI-Mass spectra.

Results: In the time spent in open arm, Fr1, and Fr3 did not show any significant effect on mice behavior, Fr2 marginally decreased the percentage of spent time by $-8.4 \%$, and Fr4 significant increased in the time spent in the open arms by $15.7 \%$. In the open arm entry number Fr1, and Fr2 did not show any significant effect on mice behavior, Fr3 marginally increased the percentage of open arm entries by $7.9 \%$, and Fr4 significantly increased the open arm entry by $18.2 \%$. The bioactive fraction (Fr.4) was subjected to more purification. Phytochemical analysis of Fr4 lead to the identification of 4,5,7-trihydroxyflavon (apigenin).

Conclusion: Semi-polar sub-fraction of S. lavandulifolia showed anxiolytic effects by increased time spent and the entry numbers in the open arms comparable to diazepam in the EPM model in mice. Bioactivity-guided isolation leads to the characterization of apigenin with flavone structure as its active constituent. Hence, it might be introduced as a new anxiolytic agent.
\end{abstract}

Implication for health policy/practice/research/medical education:

In this research bioactivity guided technique was successively used for separation of active fraction of Stachys lavandulifolia. Apigenin was the main component identified in the most active fraction suggested to be partly responsible of $S$. lavandulifolia reported effects on anxiety in traditional medicine. Hence, it might be used as antianxiety medication.

Please cite this paper as: Rabbani M, Sajjadi SE, Karimi-Firouzjaei M, Ghanadian M. Bioactivity guided isolation of apigenin from Stachys lavandulifolia Vahl. in mice with anxiolytic effects. J Herbmed Pharmacol. 2018;7(2):74-78. doi: 10.15171/jhp.2018.13.

\section{Introduction}

Anxiety disorders are among the most prevalent of all mental disorders that exist in many forms and have a huge impact on the quality of life. Anxiety is a basic symptom of many psychiatric disorders and almost inevitable component of many medical and surgical conditions. Common treatment includes serotonin specific reuptake inhibitors (SSRIs), benzodiazepines, antidepressants and mono-amine oxidase inhibitors (MAOIs) which may cause important side effects like sedation, addiction, tachycardia, insomnia, decreased libido and ineffectiveness with their long-term use (1). To overcome the side effects of the synthetic drugs, researches have focused on medicinal plants like Valeriana officinalis (2), Matricaria recutita (3), Passifelora incarnata (4), Nepeta persica (5), Tilia americana (6), Tilia europaea (7) and Stachys lavandulifolia (8). Among them S. lavandulifolia Vahl. is an herbaceous wild plant native to Iran (9) which 
is used in Iranian folk medicine as a mild sedative tea for reducing anxiety and for treatment of gastrointestinal disorders (10). In previous studies by the same authors on hydroalcohol total extract, ethyl acetate, petroleum ether, n-butanol and aqueous partitions of this plant, ethyl acetate partition showed suitable effects on anxiety EPM test in mice (11). Therefore, in the current study a possible anxiolytic property of subfractions of ethyl acetate extract of S. lavandulifolia was evaluated and the bioactive fraction was subjected to purification to found the main constituent responsible for observed effects. For anxiety test, the elevated plus-maze was done which one of the most extensively used model for the investigation of drug effects on anxiety-related behavior in mice. It is based on the aversion and fear of mice for open spaces and is done on an elevated plus-maze with two closed and two opened arms. Anxiolytics increase the entry numbers and time spent on the open arms (12). The present study was aimed to investigate the anxiolytic effects of sub-fractions of ethyl acetate extract of S. lavandulifolia on elevated plus-maze (EPM) model of anxiety and investigating the main constituent responsible for the observed effects.

\section{Materials and Methods}

Drugs and solutions

Solidified ethyl acetate extract or fractions (Fr.1-Fr.4) were weighed and prepared as $50 \mathrm{mg} / \mathrm{mL}$ stock solution in water using a trace amount of Tween 80. Diazepam (Sobhan Pharmaceutical Co., Iran) as standard drug was prepared as $0.5 \mathrm{mg} / \mathrm{mL}$ stock solution in distilled water and a trace amount of Tween 80 . Vehicle (distilled water and a trace amount of Tween 80) was used as control. Unless stated, all the chemicals were purchased from Merck Company (Germany).

\section{Plant material}

The aerial parts of S. lavandulifolia were collected in April 2013 from Sharekord (Iran). The plant was identified and the voucher specimen (No: 1113) was deposited at the Herbarium unit of the Faculty of Pharmacy, Isfahan University of Medical Sciences, Isfahan, Iran.

\section{Extraction and preliminary fractionation}

The air dried plant material powder $(1 \mathrm{~kg})$ was macerated with $6 \mathrm{~L}$ ethyl acetate $(72 \mathrm{~h} \times 4$ times $)$ at room temperature and concentrated under reduced pressure (25.4 g). Ethyl acetate extract was applied on MPLC over RP-18 silica-gel cartridge $(20 \times 4 \mathrm{~cm})$ using $\mathrm{MeOH}: \mathrm{H}_{2} \mathrm{O}(7: 3)$ as a solvent to remove chlorophylls and fats. Defatted fraction was fractionated by chromatography on an open glass column (7 X $80 \mathrm{~cm}$ ) using normal silica gel $(63-200 \mu \mathrm{m}, 400 \mathrm{~g})$ as adsorbent and mixtures of hexane: acetone as solvent to yield four fractions (Fr1, 90: 10; Fr2, 80: 20; Fr3, 70 30; Fr4, 50:50). The fractions were concentrated to dryness and stored at $0^{\circ} \mathrm{C}$ until use.
Animals

Male Syrian mice weighing 25-35 g were housed in a cage with controlled room temperature at $22-25^{\circ} \mathrm{C}$. Food and water were available. All experiments were carried out between 09:00 and 13:00. Each mouse received a single subcutaneous injection of drug or vehicle and was tested once in the EPM.

\section{Elevated plus-maze test}

The EPM test has been described in details elsewhere $(11,2)$. Briefly, the apparatus comprised of two open arms $(35 \times 5 \mathrm{~cm})$ and two closed arms $(30 \times 5 \times 15 \mathrm{~cm})$ that extended from a common central platform $(5 \times 5 \mathrm{~cm})$. The floor and the walls of each arm were wooden and painted black. The entire maze was elevated to a height of $60 \mathrm{~cm}$ above floor level as validated and described by Rabbani et al (5). Testing was conducted in a quiet room that was illuminated only by a dim light. Mice were given a single sc dose of the sub-fraction of extract 30 minutes before their placement on the EPM. To begin a test session, mice were placed on the open arm facing the open arm of the maze. An entry into an arm was defined as the animal placing all four paws over the line marking that area. The number of entries and the time spent in the open and closed arms and the number of entries in the middle arm was recorded during a 5-minute test period. The percentage of open arm entries $(100 \times$ open/total entries) was calculated for each animal. Between each trial, the elevated plus-maze was wiped clean with an alcohol (70\%) and dried with napkin.

Isolation of bioactive fraction

Antianxiety effects of the resulted fractions: Fr.1 to Fr.4 were compared in vivo on EPM model in mice. According to the EPM pharmacological results, the bioactive fraction (Fr.4) was subjected to more purification. Based on primary identification by thin layer chromatography (TLC) analyses using cerium sulfate (1\%) and natural product reagent which was applied on a SC6 polyamide column ( 2 X 20) using $\mathrm{CHCl}_{3}: \mathrm{MeOH}$ in a stepwise gradient manner $(2 \rightarrow 20 \%)$ as solvent. The major subfraction which was eluted by $\mathrm{CHCl}_{3}: \underline{\mathrm{MeOH}}(88: \underline{12})$, was purified on sephadex-LH 20 gel chromatography (2 X 60) using methanol as solvent and yielded compounds $\mathbf{1}$ (56 $\mathrm{mg}$ ) as pure compound.

Statistical analysis

Statistical analysis was performed using one-way ANOVA with post hoc Duncan test. $P<0.05$ was considered significant. All data were expressed as mean \pm S.D.

\section{Results}

Ethyl acetate extract was fractionated on column chromatography and yielded four fractions. Antianxiety effects of the resulted fractions: Fr.1 to Fr. 4 were compared 
in vivo on EPM model in mice.

\section{Elevated plus-maze}

In the time spent in open arm in EPM test in comparison to control group, diazepam group significantly increased the percentage of spent time on open arms by $39.1 \%$ $(P<0.05)$, ethyl acetate extract, Fr1, and Fr3 did not show significant effect on mice behavior, Fr2 marginally decreased the percentage of spent time by $-8.4 \%(P<0.1)$, and Fr4 had significant increase in the time spent in the open arms by $15.7 \%(P<0.05$; Figure $1 \mathrm{~A})$.

In the open arm entry number in EPM test in comparison to control group, diazepam group was marginally significant by $6.9 \%(P<0.1)$, ethyl acetate extract, Fr1, and Fr2 did not show any significant effect on mice behavior, Fr3 marginally increased the percentage of open arm entries by $7.9 \%(P<0.1)$, and Fr4 significantly increased the open arm entry by $18.2 \%(P<0.01)$ (Figure $1 \mathrm{~B})$.

The summarized data in Table 1 represent the occupancy time as well as the entry numbers into the closed arms. None of the tested fractions or diazepam showed significant change versus control $(P<0.05$, Table 1$)$.

Analysis of bioactive fraction

In between group analysis, the most bioactive fraction
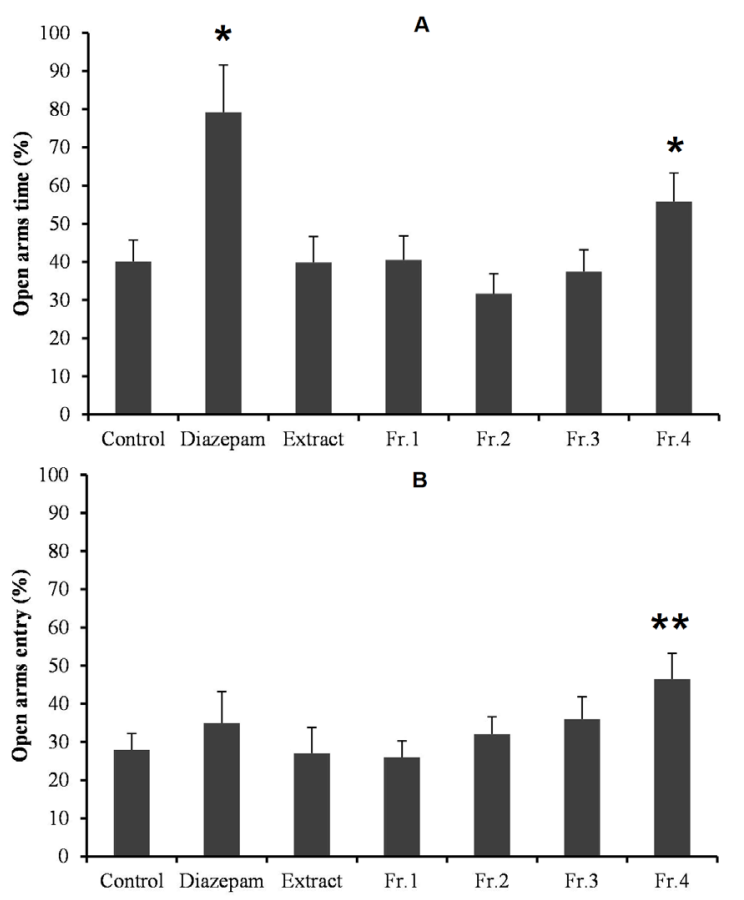

Figure 1. Effect of diazepam, ethyl acetate extract and fractions: Fr.1 - 4 of Stachys lavandulifolia on (A) the percentage of time spent in the open arms, (B) the percentage of open arm entries of the elevated plus-maze during 5 minutes test in mice. Extract, fractions (each $50 \mathrm{mg} / \mathrm{kg}$ ), diazepam $(0.5 \mathrm{mg} / \mathrm{kg}$ ), and vehicle (water and trace amount of tween 80 ) as control were injected single subcutaneous 30 minutes prior to test. Data are presented as mean values ( \pm SEM $) .{ }^{*} P<0.05,{ }^{* *} P<0.01$ compared with vehicle-treated control.
(Fr4) with more time spent and entry number percentages were selected and subjected to purification methods using polyamide and sephadex gel chromatography. Finally, it yielded a pale yellowish solid with positive reaction to natural product reagent ( $1 \%$ methanolic diphenylboric acid-ethylamino ester) and $\mathrm{FeCl}_{3}$ test. Its UV spectrum showed two absorptions maxima at 340.7 and 265.5 $\mathrm{nm}$ which is characteristic for flavones. Its ${ }^{1} \mathrm{H}-\mathrm{NMR}$ resonances at $\delta_{\mathrm{H}}(400 \mathrm{MHz}$, pyridine-d6): $6.11(1 \mathrm{H}, \mathrm{d}$, $J=2 \mathrm{~Hz}, \mathrm{H}-6), 6.37(1 \mathrm{H}, \mathrm{d}, J=2 \mathrm{~Hz}, \mathrm{H}-8), 6.50(1 \mathrm{H}, \mathrm{s}$, $\mathrm{H}-3,6.84\left(2 \mathrm{H}, \mathrm{d}, J=8.5 \mathrm{~Hz}, \mathrm{H}-5^{\prime}, 3^{\prime}\right)$ and $7.76(2 \mathrm{H}, \mathrm{d}, J$ $\left.=8.5 \mathrm{~Hz}, \mathrm{H}-5{ }^{\prime}, 3^{\prime}\right)$ indicated that isolated compound was a flavone derivative. Its negative ESI MS $m / z 269$ [M1] and comparison of ${ }^{1} \mathrm{H}$ - and ${ }^{13} \mathrm{C}-\mathrm{NMR}$ data $\left[\delta_{\mathrm{C}}(100\right.$ $\mathrm{MHz}$, pyridine-d6): $163.8,104.4,183.2,106.5,163.2$, $100.5,166.4,95.3,159.0,122.8,129.4,117.3,163.3,117.3$, 122.8] with literature and co-TLC with authenticated standards determined it as 4,5,7-trihydroxyflavon known as apigenin (13). Phytochemical analysis of other fractions (Fr1-Fr3, and Fr5) was also performed in another research work which its data were published elsewhere (14).

\section{Discussion}

In our previous studies we showed that different extracts (petroleum ether, ethyl acetate, butanol and water) of S. lavandulifolia had various degrees of anxiolytic properties (11). From these extracts, ethyl acetate proved to be more suitable for further studies as it had fewer sedative properties with acceptable anxiolytic effects. In this study, in continuing our previous studies, the ethyl acetate extract was obtained and fractionated by column chromatography. Yielded fractions (Fr1-4) at $50 \mathrm{mg} / \mathrm{kg}$ were tested again by elevated plus-maze test from which Fr4 significantly increased time spent and the entry numbers in the open arms. Analysis of the bioactive fraction, Fr4, characterized its major component as apigenin suggested

Table 1. Behavioral parameters recorded in the plus-maze from mice treated with various fractions of S. lavandulifolia

\begin{tabular}{lll}
\hline Treatment & Closed arm times & Closed arm entries (\%) \\
\hline Control & $59.9 \pm 8.4$ & $71.8 \pm 10.8$ \\
Diazepam $(0.5 \mathrm{mg} / \mathrm{kg})$ & $20.8 \pm 3.3$ & $64.9 \pm 15.2^{*}$ \\
Ethyl acetate extraction & $60.2 \pm 10.3$ & $72.6 \pm 18.4$ \\
$(50 \mathrm{mg} / \mathrm{kg})$ & $59.5 \pm 9.3$ & $63.6 \pm 12.2$ \\
FR1 $(50 \mathrm{mg} / \mathrm{kg})$ & $68.3 \pm 11.2$ & $67.3 \pm 9.8$ \\
FR2 $(50 \mathrm{mg} / \mathrm{kg})$ & $62.6 \pm 9.7$ & $63.9 \pm 10.3$ \\
FR3 $(50 \mathrm{mg} / \mathrm{kg})$ & $44.2 \pm 5.9$ & $53.6 \pm 7.8$ \\
FR4 $(50 \mathrm{mg} / \mathrm{kg})$ & &
\end{tabular}

Effects of diazepam, ethyl acetate extract and ethyl acetate extract subfractions (FR1-4) of S. lavandulifolia on the percentage of time spent in the closed arms and the closed arm entries of the elevated plus-maze during a 5-minute test in mice. Fractions (each $50 \mathrm{mg} / \mathrm{kg}$ ), diazepam $(0.5 \mathrm{mg} / \mathrm{kg})$, and control (water and trace amount of tween 80$)$, were injected single subcutaneous 30 minutes prior to test.

Data are presented as mean values $( \pm$ SEM) from a group of six mice. * $(P<0.05)$ compared with vehicle-treated control. 
to be responsible for observed anxiolytic effects. These results were in agreement with other studies by Kumar et al who showed that apigenin and apigenin-7-glucoside isolated from Stachys tibetica significantly increased the percentage of time spent and arm entries in the open arms of EPM test in mice (15). Another study conducted by Gazola et al, showed that apigenin as the main constituent of Passiflora quadrangularis pericarp induced sedative effects. They showed that apigenin sedative effect was blocked by flumazenil as benzodiazepine antagonist and therefore suggested that apigenin was doing its effect through binding to inhibitory gama amino-butyric acid (GABA) receptor or due to an enhancement of the GABAergic system (16). There is more evidence on flavonoids to act as GABAA modulators in the brain. Vaishnav et al reported that flavonoids could pass through the blood brain barrier $(\mathrm{BBB})$ and bind to the benzodiazepine GABA-A receptor site. They showed that the planar rigid shape of benzopyrone part of flavonoids made them susceptible to anxiolytic properties through GABA-A receptor benzodiazepin binding site (17). However, flavonoids still do not play a major role in the treatment of anxiety disorder. The low permeability and passage rate from $\mathrm{BBB}$ due to their hydrophilic structure require higher doses for their efficacy which increase adverse effects. It creates limitations in the effectiveness and use of these compounds in the treatment of anxiety and insomnia. To fix the problem semi-synthetic approach on their structure to increase their binding to GABA receptors and their permeability from $\mathrm{BBB}$ is required.

\section{Conclusion}

In this research bioactivity guided technique was successively used for separation of active fraction of Stachys lavandulifolia. Apigenin was the main component identified in the most active fraction suggested to be partly responsible for $S$. lavandulifolia reported effects on anxiety in traditional medicine. Hence, it might be used as antianxiety medication.

\section{Acknowledgements}

This paper is part of the thesis of Masoumeh Karimi Firouzjaei submitted in partial fulfillment of the requirements for the degree of Pharm $\mathrm{D}$. We are grateful to the Isfahan Pharmaceutical Sciences Research Center, Pharmacy faculty, Isfahan University of Medical Sciences, Isfahan, I.R. Iran for their support.

\section{Authors' contributions}

MR participated in pharmacological experiments and writing the manuscript. SES coordinated the pharmacognosy part of the study. MKF participated in most parts of the study. MG contributed to pharmacognosy part and finalizing the manuscript. All authors read and confirmed final version and publication of the manuscript.

\section{Conflict of interests}

The authors have no conflicts of interest.

\section{Ethical considerations}

The study was approved by the Ethical Committee of Isfahan University of Medical Sciences, Isfahan, Iran (393148). Ethical issues (including plagiarism, misconduct, data fabrication, falsification, double publication or submission, redundancy) have been completely observed by the authors.

\section{Funding/Support}

This project has been supported by Isfahan Pharmaceutical Sciences Research Center, Isfahan University of Medical Sciences (Grant No. 393148).

\section{References}

1. Ravindran LN, Stein MB. The pharmacologic treatment of anxiety disorders: a review of progress. J Clin Psychiatry. 2010;71(7):839-54. doi: 10.4088/JCP.10r06218blu.

2. Murphy K, Kubin ZJ, Shepherd JN, Ettinger RH. Valeriana officinalis root extracts have potent anxiolytic effects in laboratory rats. Phytomedicine. 2010;17(8-9):674-8. doi: 10.1016/j.phymed.2009.10.020.

3. Tabari MA, Tehrani MAB. Evidence for the involvement of the GABAergic, but not serotonergic transmission in the anxiolytic-like effect of bisabolol in the mouse elevated plus maze. Naunyn Schmiedebergs Arch Pharmacol. 2017;390(10):1041-6. doi: 10.1007/s00210-017-1405-0.

4. Sampath C, Holbik M, Krenn L, Butterweck V. Anxiolytic effects of fractions obtained from Passiflora incarnata L. in the elevated plus maze in mice. Phytother Res. 2011;25(6):789-95. doi: 10.1002/ptr.3332.

5. Rabbani M, Sajjadi SE, Mohammadi A. Evaluation of the anxiolytic effect of Nepeta persica Boiss. in mice. Evid Based Complement Alternat Med. 2008;5(2):181-6. doi: 10.1093/ ecam/nem017.

6. Nogueron-Merino MC, Jimenez-Ferrer E, RomanRamos R, Zamilpa A, Tortoriello J, Herrera-Ruiz M. Interactions of a standardized flavonoid fraction from Tilia americana with Serotoninergic drugs in elevated plus maze. J Ethnopharmacol. 2015;164:319-27. doi: 10.1016/j. jep.2015.01.029.

7. Coleta M, Campos MG, Cotrim MD, Proenca da Cunha A. Comparative evaluation of Melissa officinalis L., Tilia europaea L., Passiflora edulis Sims. and Hypericum perforatum L. in the elevated plus maze anxiety test. Pharmacopsychiatry. 2001;34 Suppl 1:S20-1. doi: 10.1055/ s-2001-15460.

8. Rabbani M, Sajjadi SE, Zarei HR. Anxiolytic effects of Stachys lavandulifolia Vahl on the elevated plus-maze model of anxiety in mice. J Ethnopharmacol. 2003;89(23):271-6. doi: 10.1016/j.jep.2003.09.008.

9. Ghahraman A. Color Atlas of Iranian Flora. Research Institute of Forests and Rangclands Publishing; 1996:3071.

10. Mozaffarian V. Identification of Medicinal and Aromatic Plants of Iran. Tehran: Farhang-e Moaser; 2013:1172

11. Rabbani M, Sajjadi SE, Jalali A. Hydroalcohol extract and fractions of Stachys lavandulifolia Vahl: effects on 
spontaneous motor activity and elevated plus-maze behaviour. Phytother Res. 2005;19(10):854-8. doi: 10.1002/ ptr. 1701.

12. Bourin M. Animal models for screening anxiolyticlike drugs: a perspective. Dialogues Clin Neurosci. 2015;17(3):295-303.

13. Sajjadi SE, Ghanadian M, Haghighi M. Isolation and Identification of Two Phenolic Compounds from a Moderately Cytotoxic Fraction of Cousinia verbascifolia Bunge. Adv Biomed Res. 2017;6:66. doi: 10.4103/22779175.190980.

14. Sajjadi SE, Ghanadian M, Rabbani M, Tahmasbi F. Isolation and identification of secondary metabolites from the aerial parts of Stachys lavandulifolia Vahl. Iran J Pharm Res.
2017;16:58-63.

15. Kumar D, Bhat ZA. Apigenin 7-glucoside from Stachys tibetica Vatke and its anxiolytic effect in rats. Phytomedicine. 2014;21(7):1010-4. doi: 10.1016/j.phymed.2013.12.001.

16. Gazola AC, Costa GM, Castellanos L, Ramos FA, Reginatto $\mathrm{FH}$, de Lima TCM, et al. Involvement of GABAergic pathway in the sedative activity of apigenin, the main flavonoid from Passiflora quadrangularis pericarp. Rev Bras Farmacogn. 2015;25(2):158-63. doi: 10.1016/j.bjp.2015.03.009.

17. Vaishnav GA, Kulkarni GK, Sankar S. In-Silico Docking of neuroactive flavones on benzodiazepine binding site of GABA-A receptor homology model. Int J Pharm Phytopharm Res. 2012;2(3):176-84. 